九州大学学術情報リポジトリ

Kyushu University Institutional Repository

\title{
CD30 ligand is a new therapeutic target for central nervous system autoimmunity
}

篠田，紘司

https://doi.org/10.15017/1500583

出版情報 : 九州大学，2014，博士（医学），課程博士 バージョン：

権利関係：やむを得ない事由により本文ファイル非公開（2） 
1 CD30 ligand is a new therapeutic target for central nervous system autoimmunity

2 Koji Shinoda ${ }^{1,2}$, Xun Sun $^{3}$, Akiko Oyamada ${ }^{1}$, Hisakata Yamada ${ }^{1}$, Hiromi Muta ${ }^{4}$, Eckhard R. Podack ${ }^{5}$

3 Jun-ichi Kira ${ }^{2}$, and Yasunobu Yoshikai ${ }^{1}$

$4{ }^{1}$ Division of Host Defense, Medical Institute of Bioregulation, Kyushu University, Fukuoka 812-8582,

5 Japan.

$6 \quad{ }^{2}$ Department of Neurology, Neurological Institute, Graduate School of Medical Sciences, Kyushu

7 University, Fukuoka 812-8582, Japan.

$8 \quad{ }^{3}$ Department of Immunology, China Medical University, Shenyang 110001, China.

$9 \quad{ }^{4}$ Department of Medicine and Bioregulatory Science, Graduate School of Medical Sciences, Kyushu

10 University, Fukuoka 812-8582, Japan.

$11{ }^{5}$ Department of Microbiology and Immunology, University of Miami, Miami, FL 33124, USA.

12 Author for Correspondence: Yasunobu Yoshikai, MD, PhD, Division of Host Defense, Medical

13 Institute of Bioregulation, Kyushu University, 3-1-1 Maidashi, Higashi-ku, Fukuoka 812-8582, Japan.

14 Tel: +81-92-642-6962; Fax: +81-92-642-6973; E-mail: yoshikai@bioreg.kyushu-u.ac.jp

15 The number of words in the title: 12

16 Total number of words in the abstract: 168 
18 Total number of figures: 6

19 Total number of color figures: 4

20 Key words: EAE, CD30 ligand, CD30, CD30-Ig

21 
22 Abstract

23 The CD30 ligand (CD30L)/CD30 axis plays a critical role in Th1 and Th17 cell differentiation.

24 However, the role in the pathogenesis of central nervous system autoimmunity remains unknown.

25 Here we show the resistance for experimental autoimmune encephalomyelitis (EAE) with markedly

26 reduced induction of antigen-specific Th1 and Th17 cells in CD30L knockout mice. Bone marrow

27 (BM) chimera experiments indicated that $\mathrm{CD} 30 \mathrm{~L}$ on BM-derived cells were critical for the

28 development of EAE and that CD30L reverse signaling in CD4 T cells was dispensable for the

29 pathogenic Th17 cell differentiation at the induction phase. Adoptive transfer experiment revealed an

30 additional role for $\mathrm{CD} 30 \mathrm{~L}$ in the environment at the effector phase. In vivo neutralization of CD30L by

31 soluble murine CD30-Immunoglobulin fusion protein before disease onset or even after disease onset

32 significantly ameliorated the clinical symptoms. These results indicate that CD30L/CD30 signaling is

33 critically involved in antigen-specific CD4 $\mathrm{T}$ cell responses at both the induction and effector phase,

34 thus could be a new target molecule for the treatment of central nervous system autoimmunity. 


\section{Introduction}

37 Multiple sclerosis (MS) is an inflammatory demyelinating disorder of the central nervous system

38 (CNS). Experimental autoimmune encephalomyelitis (EAE) is an animal model of MS, which is

39 induced actively by immunization with myelin antigen $(\mathrm{Ag})$ or passively by adoptive transfer of myelin-specific CD4 T cells into naive mice [1, 2]. Both Th1 and Th17 cells were shown to induce

41 EAE, as Th1 cells might promote the entry of Th17 to the CNS during EAE [3, 4]. We have recently reported that tyrosine kinase 2 (Tyk2), which is involved in interleukin (IL)-12-signaling for Th1 cell differentiation and IL-23-signaling for Th17 cell differentiation, plays an indispensable role in the development of EAE [5]. Thus, both IL-12/Th1 and IL-23/Th17 axis might be involved in the pathogenesis of EAE. CD30 ligand (CD30L, CD153, TNFSF8) is a 40-kDa type II membrane-associated glycoprotein belonging to the tumor necrosis factor (TNF) superfamily (TNFSF) [6], and is expressed on macrophages, dendrocytes (DCs), $\mathrm{CD}^{+} \mathrm{CD} 3^{-} \mathrm{CD} 11 \mathrm{c}^{-}$accessory cells, $\mathrm{B}$ cells, activated $\mathrm{CD} 4 \mathrm{~T}$ cells, and $\gamma \delta$ T cells [7-10]. CD30 (TNFRSF8), the receptor for CD30L and a member of the TNF receptor super family (TNFRSF), is a $120 \mathrm{kDa}$ type I membrane associated glycoprotein and preferentially expressed on activated or memory helper T cells but not on resting cells [11, 12]. CD30 binds several 
53 TRAF2 [13, 14]. As for other TNFSF/TNFRSF members, reverse signaling via CD30L has been

54 reported $[7,15,16]$. In the past, $\mathrm{CD} 30 \mathrm{~L} / \mathrm{CD} 30$ signaling was thought to be preferentially involved in

55 Th2 cell responses [17-19]. However, we have revealed that CD30L/CD30 axis was involved in both

56 Th1 and Th2 cell responses [20-22], and regulatory T cells were reported to suppress allograft

57 rejection via a CD30-dependent mechanism [23, 24]. Furthermore, we recently found that the

$58 \mathrm{CD} 30 \mathrm{~L} / \mathrm{CD} 30$ axis had a critical role in Th17 differentiation in vitro and in vivo [16, 25]. Thus,

59 CD30L/CD30 signaling may not be linked to a commitment step for a particular subset differentiation,

60 but might promote cell survival of pre-activated T cells, resulting in an acceleration of various helper

61 T cell responses.

62 In the present study, we found that CD30L knockout (KO) mice were resistant to myelin

63 oligodendrocyte glycoprotein (MOG) $)_{35-55}$ peptide-induced EAE. The numbers of $\mathrm{MOG}_{35-55}$

64 peptide-specific Th1 and Th17 cells in the draining lymph nodes (dLNs) were markedly reduced in

65 CD30L KO mice as compared with wild type (WT) mice. CD30L/CD30 signaling on CD4 T cells

66 promoted Ag-specific CD4 $\mathrm{T}$ cell responses at the induction phase, and CD30L in the environment

67 was important at the effector phase in mice developing EAE. In vivo neutralization of CD30L by

68 soluble murine CD30-immunoglobulin (mCD30-Ig) fusion protein significantly ameliorated EAE 
70 the CD30L/CD30 axis is critically involved in pathogenic $\mathrm{T}$ cell responses not only in dLNs at the

71 induction phase but also in spinal cords at the effector phase, and thus modulation of CD30L/CD30

72 signaling could be a new biological therapy for the treatment of MS and other inflammatory CNS

73 demyelinating diseases.

74 
75

76

77

78

79

80

81

82

83

84

85

86

87

88

89

90

91

\section{Materials and methods}

2.1. Mice

C57BL/6 mice were purchased from Japan KBT (Shizuoka, Japan). CD30L KO mice were generated as previously described [26, 27]. All mice were maintained under specific-pathogen free conditions at our institute and were backcrossed with C57BL/6 mice at least eight times. All mice were used at 8-12 weeks of age. All experiments were approved by the Committee of Ethics on Animal Experiments in the Faculty of Medicine, Kyushu University.

\subsection{Antibodies and reagents}

MOG $_{35-55}$ (MEVGWYRSPFSRVVHLYRNGK) peptide was purchased from Medical \& Biological

Laboratories (Nagoya, Japan). Fc $\gamma$ receptor blocking monoclonal antibody (mAb) (anti-CD16/32,

2.4G2), allophycocyanin (APC) and APC-H7-conjugated anti-CD4 (RM4-5), APC-conjugated CD45.2

(104), FITC-conjugated anti-CD45.1 (A20), V450 and FITC-conjugated anti-CD11b (M1/70),

FITC-conjugated anti-Foxp3 (FJK-16s), PerCP-Cy5.5-conjugated anti-major histocompatibility

complex (MHC) class II (M5/144.15.2), PE-conjugated anti-CD30 (CD30.1), PE-conjugated

anti-CD30L (RM153), PE-conjugated anti-CD154 (MR1), PE-conjugated anti-Gr-1 (RB6-8C5),

PE-Cy7-conjugated anti-IFN- $\gamma$ (XMG1.2), Alexa Flour 647-conjugated anti-CC chemokine receptor

(CCR) 6 (140706) mAbs were purchased from BD Biosciences (San Diego, CA). Alexa Flour 
647-conjugated anti-CD3 (17A2), Alexa Flour 647-conjugated IL-17A (TC11-18H10) mAb, rat IgG2b

93 was purchased from e-Bioscience (San Diego, CA). Recombinant mouse IL-23 was purchased from

$94 \quad$ R\&D Systems (Minneapolis, MN).

\subsection{Active EAE}

Female mice at 8-12 weeks of age were subcutaneously (s.c.) immunized with $200 \mu \mathrm{g}$ of $\mathrm{MOG}_{35-55}$

peptide emulsified with complete Freund's adjuvant (CFA) containing $500 \mu \mathrm{g}$ of Mycobacterium

tuberculosis H37RA (BD Biosciences) on day 0 and were i.p. injected with $500 \mu \mathrm{g}$ of pertussis toxin

99 (PTX) (List Biological Laboratories, Campbell, CA) on day 0 and 2 [1]. Clinical symptoms were

100 scored according to the following criteria: 0 , unaffected; 1 , flaccid tail; 2, impaired gait; 3, partial hind

101 limb paralysis; 4, complete hind limb paralysis; 5, total hind limb paralysis with partial forelimb

102 paralysis [28].

103

\subsection{Passive EAE}

104 Donor mice were s.c. immunized with $200 \mu \mathrm{g}$ of $\mathrm{MOG}_{35-55}$ peptide emulsified with CFA. Ten days

105 later, dLNs were harvested and single-cell suspensions were incubated in the presence of $10 \mu \mathrm{g} / \mathrm{ml} \mathrm{of}$

$106 \mathrm{MOG}_{35-55}$ peptide and $10 \mathrm{ng} / \mathrm{ml}$ of recombinant $\mathrm{IL}-23$ in a $37^{\circ} \mathrm{C}, 5 \% \mathrm{CO}_{2}$ incubator for 72 hours.

107 Recipient mice were irradiated with 4 Gy and intravenously transferred with $2 \times 10^{7}$ cultured cells on 
110 Whole dLNs cells were cultured in 96-well plates with $10 \mu \mathrm{g} / \mathrm{ml}$ of $\mathrm{MOG}_{35-55}$ peptide for 72 hours.

111 Wells were pulsed with ${ }^{3} \mathrm{H}$-thymidine (New England Nuclear, Boston, MA) at $37 \mathrm{mBq} /$ well for the

112 final 12 hours. Mean incorporation was measured by a 1205 Betaplate scintillation counter

113 (PerkinElmer, Gaithersburg, MD).

114 2.6. Isolation of mononuclear cells from spinal cords

115 Spinal cords were excised from mice perfused transcardially with ice-cold phosphate buffered saline

116 (PBS). They were cut into small pieces and digested with collagenase D (Invitrogen, Carlsbad, CA)

117 and DNase I (DN25; Sigma-Aldrich, Tokyo, Japan). Dispersed cells were passed through a nylon

118 mesh, placed onto 33\% Percoll solution (GE Healthcare, Buckinghamshire, UK), and centrifuged at

$119800 \times g$ for 20 minutes. Cell pellets were suspended with culture medium and used for analysis.

120 2.7. Flow cytometric analysis and intracellular staining

121 For cell surface staining, single-cell suspensions were incubated with an optimal concentration of

122 fluorescent Abs in Hanks' Balanced Salt Solution with $0.5 \%$ fetal bovine serum for 20 minutes at $4^{\circ} \mathrm{C}$.

123 Intracellular staining was performed using the BD Cytofix/Cytoperm kit (BD Biosciences) according

124 to the manufacturer's instructions. In some experiments, we added propidium iodide $(1 \mu \mathrm{g} / \mathrm{ml})$ to cell

125 suspensions just before running on a flow cytometer to detect and exclude dead cells for the analysis. 
126 Stained cells were run on a FACS Calibur or FACS Verse flow cytometer (BD Biosciences). The data

127 were analyzed using FlowJo software (TreeStar, San Carlos, CA).

128 2.8. Measurement of $M O G_{35-55}$ peptide-specific cytokine production

129 Single cell suspensions were harvested from dLNs of EAE induced mice at day 9. Whole dLNs cells

130 were cultured in 96-well plates with $10 \mu \mathrm{g} / \mathrm{ml}$ of $\mathrm{MOG}_{35-55}$ peptide for 72 hours. The amount of

131 cytokines in the culture supernatants was measured by DuoSet ELISA Development System (R\&D

132 Systems). For intracellular staining, single-cell suspension from dLNs or spinal cords were incubated

133 for 5 hours with $25 \mu \mathrm{g} / \mathrm{ml}$ of $\mathrm{MOG}_{35-55}$ peptide in the presence of $10 \mu \mathrm{g} / \mathrm{ml}$ of brefeldin $\mathrm{A}$

134 (Sigma-Aldrich) for the last 4 hours.

135 2.9. Histopathological analysis

136 Mice were transcardially perfused with PBS and 4\% paraformaldehyde in 0.1 M PBS. Spinal cords

137 were excised and embedded in optimal cutting temperature compound and sectioned horizontally on a

138 cryostat. Sections were stained by hematoxilin and eosin (H\&E) and Klüver-Barrera's staining.

139 2.10. Generation of bone marrow chimera

140 Bone marrow (BM) cells were extracted from WT (Ly5.1/5.1) and CD30L KO (Ly5.2/5.2) mice by

141 flushing the femurs and tibias and were then depleted of T cells using anti-CD3 mAb (17A2,

142 eBioscience) and Dynabeads sheep anti-rat IgG (Invitrogen). Red blood cells were lysed with $0.83 \%$ 
143 ammonium chloride. Remaining cells were intravenously injected into lethally (10 Gy) irradiated

144 recipient mice. For mixed BM chimeras, BM cells from WT (Ly5.1/5.1) and CD30L KO (Ly5.2/5.2)

145 were mixed at a ratio of 1:1 and were intravenously injected into lethally irradiated recipient mice

146 (Ly5.1/5.2). Eight weeks later, BM reconstitution was confirmed and EAE was induced.

147 2.11. Generation and in vivo administration of $\mathrm{mCD} 30-\mathrm{Ig}$

148 A soluble mCD30-Ig fusion protein was obtained as previously described [25, 29]. mCD30-Ig fusion

149 protein cDNA was expressed by the vector pBMGNeo in NIH 3T3 cells. Secreted mCD30-Ig protein

150 from growing NIH 3T3 cells in serum-free medium (SFM101; Nissui Pharmaceutical, Tokyo, Japan)

151 was purified by HiTrap Protein G HP (GE Healthcare) and analyzed by SDS-PAGE for purity.

$152 \mathrm{mCD} 30-\mathrm{Ig}$, diluted at a concentration of $1 \mathrm{mg} / \mathrm{mL}$ in PBS, was stored $-80^{\circ} \mathrm{C}$ until use. For in vivo

153 neutralization, $200 \mu \mathrm{g}$ of mCD30-Ig or PBS was i.p. injected into recipient mice.

154 2.12. Statistical analysis

155 Statistical significance was evaluated by Student's $t$-test and Dunnett's test using JMP9 software (SAS,

156 Cary, NC). Differences with $p$ values $<0.05$ were considered statistically significant. 
158

159

160

161

162

163

164

165

EAE.

\section{Results}

3.1. CD3OL KO mice are resistant to active EAE.

We examined the role of the CD30L/CD30 axis in the pathogenesis of active EAE induced by

immunization of mice with $\mathrm{MOG}_{35-55}$ peptide emulsified with CFA, followed by intraperitoneal (i.p.)

injection with PTX on days 0 and 2. WT mice started to develop EAE about 10 days after

immunization and reached a peak score at about 3 weeks with $100 \%$ incidence, whereas clinical

symptoms were significantly ameliorated in CD30L KO mice when compared with WT mice (Fig.

1A). Histological analysis of spinal cords was performed 18 days and 40 days after immunization for

EAE induction. Inflammatory cell infiltration was reduced in CD30L KO mice compared with WT

mice at the peak disease phase (Fig. 1B), and the severity of demyelination was attenuated in CD30L

KO mice at the chronic phase (Fig. 1C). To quantify the extent of cellular infiltration, flow cytometric analysis was performed and revealed significantly impaired infiltration of CD4 ${ }^{+} \mathrm{MHC}$ Class $\mathrm{II}^{-}(\mathrm{CD} 4 \mathrm{~T}$ cell), CD11 b $\mathrm{bHC}^{+}$Class $\mathrm{II}^{+}$(macrophage/microglia) and Gr- $1^{+} \mathrm{MHC}$ Class $\mathrm{II}^{-}$(granulocyte) cells in CD30L KO mice (Fig. 1D). Thus, the CD30L/CD30 axis is involved in the development of active 
176 To examine the role of the CD30L/CD30 axis in the development of $\mathrm{MOG}_{35-55}$ peptide-specific CD4 T

177 cells, dLNs were harvested 9 days after immunization and were restimulated in vitro with or without

178 various concentrations of $\mathrm{MOG}_{35-55}$ peptide. $\mathrm{Ag}$-specific $\mathrm{T}$ cell proliferation was assessed by

179 thymidine incorporation assay. Thymidine uptake was significantly reduced in CD30L KO mice

180 compared with WT mice (Fig. 2A). Ag-specific T cells are reported to be defined by intracellular

181 staining of CD154 after in vitro stimulation with $\mathrm{Ag}$ [30]. As shown in Fig. 2B, the frequency and

182 number of $\mathrm{CD} 154^{+} \mathrm{CD} 4 \mathrm{~T}$ cells in dLNs was significantly lower in CD30L KO mice compared with

183 WT mice. Furthermore, intracellular cytokine staining of a cell population gated on CD4+ T cells after

184 restimulation with $\mathrm{MOG}_{35-55}$ peptide revealed that CD30L KO mice had significantly reduced levels of

$185 \mathrm{MOG}_{35-55}$ peptide-specific Th1 and Th17 cells during the development of EAE (Fig. 2C). However,

186 there were no differences in the percentages and cell numbers of Forkhead box p3 (Foxp3) ${ }^{+}$regulatory

187 CD4 T cells in dLNs between WT and CD30L KO mice (Fig. S1). We further examined cytokine

188 production from dLNs cells harvested on day 9 after immunization by enzyme-linked immunosorbent

189 assay (ELISA) after 3 days culture in the presence of $\mathrm{MOG}_{35-55}$ peptide. Consistent with flow

190 cytometric analysis, dLNs cells from CD30L KO mice produced significantly attenuated levels of

191 IL-17 and interferon (IFN)- $\gamma$ (Fig. 2D), whereas there were no differences in IL-2, IL-4, IL-10 and 
192 TNF- $\alpha$ production between WT and CD30L KO mice (data not shown).

193 We previously reported that $\mathrm{CD} 30^{+} \mathrm{T}-\mathrm{CD} 30 \mathrm{~L}^{+} \mathrm{T}$ cell interactions mediated $\mathrm{Th} 1$ cell responses in

194 Mycobacterium bovis Bacillus Calmette-Guérin (BCG) infection, and played a critical role in Th17

195 cell differentiation in virto, while CD30L expression on Ag-presenting cells (APCs) was dispensable

196 in the development of Ag-specific Th1 and Th17 cells [16, 21]. To determine which cells are involved

197 in mice developing EAE, we examined the expression pattern of CD30L. As previously described[8],

198 CD30L was expressed on CD4 T cells, CD8 T cells, macrophages and DCs (Fig. S2). Unique

$199 \mathrm{CD}^{+} \mathrm{CD}^{-} \mathrm{CD} 11 \mathrm{c}^{-}$accessory cells were reported to be the constitutive source of CD30L [9]. However,

200 we could not detect its expression. Subsequently, we cultured dLNs cells in a 96-well plate containing

201 medium with $\mathrm{MOG}_{35-55}$ peptide $(10 \mu \mathrm{g} / \mathrm{ml})$, or in a plate coated by anti-CD3 mAb $(5 \mu \mathrm{g} / \mathrm{ml})$ for 24

202 hours, and examined CD30L expression. CD30L expression was enhanced by stimulation with

$203 \mathrm{MOG}_{35-55}$ peptide, and almost all CD4 $\mathrm{T}$ cells expressed CD30L after stimulation by anti-CD3 mAb

204 (Fig. S3). To address the possibility that CD30L on APCs is important for Ag presentation to CD4 T

205 cells, we purified CD4 T cells from dLNs on day 9 after immunization and co-cultured them with 30

206 Gy-irradiated splenocytes from WT and CD30L KO mice as APCs in the presence of $\mathrm{MOG}_{35-55}$

207 peptide for 72 hours. There were no differences in IL-17 and IFN- $\gamma$ production in the culture

208 supernatant as measured by ELISA between WT and CD30L KO APCs (Fig. 2E). Thus, CD30L 
expression on APCs is dispensable during Ag presentation to primed CD4 T cells to induce IL-17 and

IFN- $\gamma$ production.

211 Furthermore, we also examined the expression levels of CCR6 on CD4 T cells at the induction phase,

212 and found that the frequency and cell number of CCR6 $6^{+} \mathrm{IL}-17 \mathrm{~A}^{+} \mathrm{CD} 4 \mathrm{~T}$ cells were significantly

213 attenuated in CD30L KO mice compared with WT mice (Fig. S4). Importantly, there were no

214 differences in the percentages of $\mathrm{CCR}^{+}$cells in Th17 cell between WT and CD30L KO mice. Thus,

215 we considered that CCR6 expression was diminished in association with impaired development of

216 Th17 cells in CD30L KO mice.

218 3.3. CD30L on hematopoietic cells and radioresistant cells was involved in EAE development

219 As previously described, CD30L was expressed on various cell types including activated CD $4^{+}$helper

220 T cells, macrophages, DCs in dLNs at the induction phase (Fig. S2). We also found an appreciable

221 level of CD30L expression on macrophages and microglial cells in addition to infiltrating CD4 T cells

222 in the spinal cord at the effector phase (Fig. S5). To elucidate the role of CD30L expression on BM

223 derived cells or radioresistant host cells in EAE development, we set up three groups of BM chimeras

224 (Fig. 3A): (1) Recipient WT (Ly5.2/5.2) mice were lethally irradiated and transferred with BM cells

225 from WT (Ly5.1/5.1) mice (WT -> WT); (2) Recipient CD30L KO (Ly5.2/5.2) mice were lethally 
226 irradiated and transferred with BM cells from WT (Ly5.1/5.1) mice (WT -> CD30L KO); and (3)

227 Recipient WT (Ly5.1/5.1) mice were lethally irradiated and transferred with BM cells from CD30L

228 KO (Ly5.2/5.2) mice (CD30L KO -> WT). After confirming BM reconstitution 8 weeks later, BM

229 chimera mice were immunized with $\mathrm{MOG}_{35-55}$ peptide emulsified with CFA. As shown in Fig. 3B and

230 C, development of EAE in CD30L KO-> WT mice were significantly ameliorated compared with WT

231 -> WT mice, confirming that CD30L expression on BM derived cells have a pivotal role in EAE

232 development. Although the severity of EAE was comparable to WT -> WT mice, it was notable that

233 the onset of EAE was significantly delayed in WT -> CD30L KO mice. These results suggest that

234 CD30L expression on BM derived cells has a critical role but its expression on radioresistant host cells

235 might at least partly be involved in EAE development.

236

237 3.4. CD30L reverse signaling in CD4 T cells is dispensable for their differentiation into Ag-specific

$238 \quad$ CD4 T cells

239 We previously reported that both CD30 and CD30L are expressed on activated helper T cells and that

240 both CD30 signaling and CD30L reverse signaling in CD4 T cells are involved in Th17 differentiation

241 [16]. To determine whether CD30L reverse signaling in CD4 T cells is involved in the development of

242 EAE, we next performed a mixed BM chimera experiment. As shown in Fig. S6A, recipient WT mice 
243 (Ly5.1/5.2) were lethally irradiated and transferred with a mixture of BM cells from WT (Ly5.1/5.1)

244 and CD30L KO (Ly5.2/5.2) mice. After confirming reconstitution 8 weeks after transfer, the chimera

245 mice were immunized with $\mathrm{MOG}_{35-55}$ peptide emulsified with CFA. The mixed BM chimera mice

246 developed mild EAE, probably due to the effect of artificial manipulation by bone marrow

247 transplantation and the relatively old week-age recipient mice (Fig. S6B). On day 10 after

248 immunization, dLNs cells were harvested and the expression of CD154, intracellular staining of

249 IL-17A and IFN- $\gamma$ in CD4 T cells were examined fter restimulation with $\mathrm{MOG}_{35-55}$ peptide for 5 hours.

250 The frequencies of CD154 ${ }^{+} \mathrm{CD} 4 \mathrm{~T}$ cells, IL-17A $\mathrm{A}^{+} \mathrm{CD} 4 \mathrm{~T}$ cells and IFN- $\gamma^{+} \mathrm{CD} 4 \mathrm{~T}$ cells in

251 CD30L-deficient CD4 T cells were comparable to those in WT CD4 T cells (Fig. S6C and D). Twenty

252 days after immunization, we examined the chimerism of CD4 T cells from dLNs and spinal cords. The

253 ratio of WT (Ly5.1/5.1) to CD30L KO (Ly5.2/5.2) CD4 T cells was similar between spinal

254 cord-infiltrating cells and dLNs cells (Fig. S6E). These results indicate that reverse signaling via

$255 \mathrm{CD} 30 \mathrm{~L}$ on $\mathrm{CD} 4 \mathrm{~T}$ cells is dispensable for their differentiation into $\mathrm{MOG}_{35-55}$ peptide-specific CD4 $\mathrm{T}$

256 cells and infiltration into the spinal cord.

258 3.5. CD30L in the environment at the effector phase is involved in EAE development and accumulation

of spinal cord inflammatory cells 
260 We detected CD30L expression in macrophage and microglia cells in addition to activated CD4 T cells

261 in the spinal cord of WT mice developing EAE (Fig. S5) and BM chimera experiments suggested that

262 CD30L expression on radioresistant host cells might at least partly be involved in the development of

263 EAE (Fig. 3B and C). To examine the requirement of CD30L in the environment, we next induced

264 passive EAE by transferring $\mathrm{MOG}_{35-55}$ peptide-primed WT CD4 T cells into naive WT and CD30L KO

265 mice. Naive CD30L KO recipients developed significantly attenuated EAE and significantly mild

266 body weight loss after receiving MOG $_{35-55}$ peptide-primed WT CD4 T cells (Fig. 4A and B).

267 Consistent with these results, histopathological examination performed 40 days after transfer revealed

268 reduced infiltration of mononuclear cells into spinal cords by H\&E staining (Fig. 4C) and reduced

269 demyelination in Klüver-Barrera's staining in CD30L KO mice (Fig. 4D). These results indicate that

$270 \mathrm{CD} 30 \mathrm{~L}$ in the environment, possibly on microglia, also plays an important role in the recruitment

271 and/or in situ activation of effector CD4 T cells in the spinal cord during the effector phase of EAE.

273 3.6. Soluble mCD30-Ig-treatment inhibits EAE development

274 We examined the effect of inhibition of CD30L/CD30 signaling by soluble mCD30-Ig fusion protein

275 in the course of active EAE in WT mice (Fig. 5A). Clinical scores and body weight loss were

276 significantly improved in mice treated with mCD30-Ig at the induction phase (Fig. 5B and C). 
277 Histological analysis performed on day 18 showed reduced infiltration of mononuclear cells into CNS

278 parenchyma (Fig. 5D). The frequency of $\mathrm{MOG}_{35-55}$ peptide-specific CD4 T cells examined by CD154

279 staining and $\mathrm{MOG}_{35-55}$ peptide-specific Th17 cells were significantly decreased in dLNs of mice

280 treated with mCD30-Ig at the induction phase (Fig. 5E and F). These results indicated that the

281 administration of soluble mCD30-Ig at the induction phase inhibited Ag-specific CD4 $\mathrm{T}$ cell responses

282 and ameliorated active EAE.

283 Our study revealed that the CD30L/CD30 axis was involved in the development of EAE both at the

284 induction phase in dLNs and at the effector phase in the spinal cord after EAE induction. Therefore,

285 we examined the effect of mCD30-Ig administered at the effector phase after disease onset (Fig. 6A).

286 Mice administered mCD30-Ig on days 12, 14, 16, 18 and 20 after disease onset had reduced clinical

287 scores and body weight loss at the chronic phase (Fig. 6B and C). Consistent with clinical symptoms,

288 histopathological analysis performed on day 40 revealed reduced infiltration of mononuclear cells and

289 apparently reduced demyelination in the spinal cords of mCD30-Ig-treated mice compared with

290 controls (Fig. 6D and E). Thus, in vivo blockade of CD30L/CD30 signaling by soluble mCD30-Ig

291 even after onset of EAE significantly protected WT mice against EAE, suggesting the therapeutic

application of CD30-Ig for MS treatment. 


\section{Discussion}

294 This study elucidated a modulatory role for CD30L/CD30 signaling in the pathogenesis of EAE. First,

295 we observed that CD30L KO mice were resistant to active EAE. At the induction phase, CD30L/CD30

296 signaling, presumably executed by T-T interactions, was involved in the differentiation of $\mathrm{MOG}_{35-55}$

297 peptide-specific Th1 and Th17 cells in dLNs. At the effector phase in spinal cord, CD30L was

expressed on macrophages and microglia as well as infiltrating CD4 T cells. Furthermore, $\mathrm{MOG}_{35-55}$

299 peptide primed T cells induced milder passive EAE in CD30L KO mice than that in WT mice,

300 suggesting that CD30L in the environment at the effector phase is also involved in the development of

301 EAE. In vivo neutralization of CD30L by soluble mCD30-Ig fusion protein ameliorated EAE in WT

302 mice when administered before and after disease onset. These results indicate that the CD30L/CD30

303 axis plays an important role in the two-step activation of CD4 T cells during the pathogenesis of EAE,

304 and thus could be a new target molecule for the treatment of MS.

305 We previously reported that CD30L/CD30 signaling executed by CD30+T-CD30L+T cell interactions

306 is involved in Th1 responses that produce IFN- $\gamma$ against BCG infection [21]. Furthermore, we recently

307 found that naïve $\mathrm{CD} 44^{+} \mathrm{CD} 44^{\text {low }} \mathrm{CD} 62^{\text {hi }} \mathrm{T}$ cells purified from $\mathrm{CD} 30 \mathrm{KO}$ or $\mathrm{CD} 30 \mathrm{~L} \mathrm{KO}$ mice with

308 C57BL/6 background exhibited impaired Th17 cell differentiation after in vitro culture under 
310 peptide-specific Th1 and Th17 cells in dLNs was greatly reduced in CD30L KO mice compared with

311 WT mice at the induction phase of EAE, and that the there was no difference in recall responses of

312 WT CD4 T cells using WT or CD30L KO APCs.

313 Similar to other members of TNFSF proteins [31], it was reported that CD30L might transmit reverse

314 signals to downregulate IL-2 production in CD4 T cells $[7,15,16]$. Because IL-2 is reported to inhibit

315 Th17 differentiation [32], CD30L reverse signaling on CD4 T cells might be also responsible at least

316 in part for differentiation of Th17 cells via down-regulation of IL-2 production. However, IL-2

317 production by CD30L-deficient CD4 $\mathrm{T}$ cells in response to $\mathrm{MOG}_{35-55}$ peptide was not significantly

318 increased in CD30L KO mice. Furthermore, mixed BM chimera experiments revealed that reverse

319 signaling was dispensable at the induction phase in mice developing EAE.

320 After priming in dLNs, effector CD4 Th1 cells producing IFN- $\gamma$ and Th17 cells producing IL-17A

321 leave dLNs and traffic through the choroid plexus into the subarachnoid space, where they encounter

322 Ag presented by meningeal APCs such as resident microglia and inflammatory DCs/macrophages/B

323 cells [33]. Consequently, the effector helper T cells are restimulated and undergo clonal expansion,

324 producing cytokines that evoke EAE. In the present study, adoptive transfer experiments revealed that

325 CD30L expression on host CNS cells was required at the effector phase in the CNS. Interestingly, BM

326 chimera experiments revealed that expression of CD30L on BM-derived donor cells played a critical 
327 role in the development of EAE, and that $\mathrm{CD} 30 \mathrm{~L}$ on radioresistant host CNS cells, presumably

328 resident microglia, also participated in the development of EAE, especially at early stages of the

329 effector phase. CD30L is expressed on various cells including activated CD4 T cells, macrophages,

330 DCs, $\mathrm{CD}^{+}{ }^{+} \mathrm{CD} 3^{-} \mathrm{CD} 11 \mathrm{c}^{-}$accessory cells, B cells, and $\gamma \delta \mathrm{T}$ cells [7-10]. We also detected CD30L

331 expression on microglia cells in addition to infiltrating activated CD4 T cells in the spinal cord of WT

332 mice developing EAE, during which processing and presentation of myelin Ag in the CNS is required

333 for initiation of disease progression [34]. Activated CD4 T cells interacts MHC Class $\mathrm{II}^{+}$APCs and are

334 reactivated in the subarachnoid space [35], and reactivation of autoreactive T cells is reported to

335 determine the severity of EAE [36]. The impaired recruitment and/or in situ reactivation of effector

336 CD4 T cells in the spinal cord at the effector phase might be partly responsible for the disease

337 resistance observed in CD30L KO mice.

338 Brain lesions in mice developing EAE preferentially express C-C Chemokine receptor (CCR) $6^{+}$

339 effector T cells recruited by C-C chemokine ligand (CCL) 20 [37]. This raises an alternative

340 possibility that $\mathrm{CD} 30 \mathrm{~L} / \mathrm{CD} 30$ signaling recruits effector $\mathrm{T}$ cell subsets to the CNS via expression of

341 chemokines on the blood brain barrier and/or chemokine receptors on T cells. As shown in Fig. S4, our

342 present results indicated that the numbers of $\mathrm{CCR} 6^{+} \mathrm{CD} 4 \mathrm{~T}$ cells were significantly reduced in $\mathrm{CD} 30 \mathrm{~L}$

343 KO mice in association with impaired development of Th17 cells. However, the Th17 cells in CD30L 
344 KO mice expressed the equivalent level of CCR6 to those in WT mice. CD30L/CD30 signaling was

345 reported to upregulate CCR7 rather than CCR6 in human YT lymphoma cell line [39]. Furthermore, it

346 was also reported to upregulate the expression of CCL21, a ligand for CCR7 in the absence of

347 lymphotoxin $\alpha$ [40]. Th17 cells are reported to express CCR6 preferentially [37]. Therefore, the

348 reduced numbers of $\mathrm{CCR}^{+} \mathrm{CD} 4 \mathrm{~T}$ cells in $\mathrm{CD} 30 \mathrm{~L}$ KO mice may be simply as a result of impaired

349 Th17 response in CD30L KO mice and not due to defect in direct signaling via CD30L/CD30

350 signaling. Further study should be required to clarify the possibility.

351 We previously reported that both Th1 and Th17 responses in lamina propria of the intestine were

352 impaired in CD30L KO mice with experimentally-induced colitis such as colitis induced by transfer of

$353 \mathrm{CD}^{+} \mathrm{CD}^{+} \mathrm{RB}^{\mathrm{hi}} \mathrm{T}$ cells in SCID mice and dextran sulphate sodium (DSS)-induced colitis in BALB/c

354 background mice [16, 25]. Soluble mCD30-Ig inhibited Th17 cell differentiation in vitro, and

355 ameliorated acute and chronic DSS-induced colitis in WT mice [25]. Thus, the modulation of

356 CD30L/CD30 signaling by soluble mCD30-Ig could be a novel biological therapy for inflammatory

357 diseases associated with Th17 responses. In the present study, mCD30-Ig treatment at the induction

358 phase ameliorated EAE by reducing the differentiation of $\mathrm{MOG}_{35-55}$ peptide-specific Th17 cells in

359 dLNs. More importantly, even if mCD30-Ig was administered after clinical disease onset, inhibition of

360 the $\mathrm{CD} 30 \mathrm{~L} / \mathrm{CD} 30$ axis resulted in milder paralysis at the chronic phase. The present study revealed 
361 that modulation of $\mathrm{CD} 30 \mathrm{~L} / \mathrm{CD} 30$ axis by soluble mCD30-Ig could be an attractive candidate for the

362 treatment of MS.

363 TNF has a complicated role in the pathogenesis of EAE and MS. TNF neutralization by anti-TNF

364 antibody ameliorated EAE [38, 39], however inhibition of TNF by mAb or TNF receptor p55

365 Immunoglobulin fusion protein exacerbated neurological symptoms of MS [40, 41]. Recently, it has

366 become clear that two TNF receptors, TNFR1 and TNFR2 have opposite roles in EAE and selective

367 inhibition of TNFR1 protected mice from EAE [42-44]. Given these complicated results, it is

368 important to investigate carefully the occurrence of side effects during clinical trials of CD30L/CD30

369 axis modulation for MS.

370

371 5. Conclusion

372 In conclusion, the present study revealed the CD30L/CD30 axis was critically involved in pathogenic

373 T cell responses not only at the induction phase in dLNs but also at the effector phase in the CNS. In

374 vivo neutralization of CD30L by soluble mCD30-Ig fusion protein ameliorated clinical symptoms of

375 EAE even when administered after disease onset. These results indicate that CD30L could be a new

376 target molecule for the treatment of MS and other inflammatory CNS demyelinating diseases. 
378 Jun-ichi Kira is an advisory board member for Merck Serono and a consultant for Biogen Idec Japan. He has received payment for lectures from Bayer Schering Pharma, Cosmic Cooperation, and Biogen Idec Japan. The other authors have declared that no conflict of interest exists.

\section{Acknowledgements}

382 This work was supported by a Grant-in-Aid from the Japan Society for the Promotion of Science, and

383 grants from the Japanese Ministry of Education, Science and Culture (Y.Y.), Yakult Bioscience

384 Foundation (Y.Y.) and Takeda Science Foundation (Y.Y.). We thank Kanako Motomura in the 385 Laboratory for Technical Support, Medical Institute of Bioregulation, and Sachiko Koyama in the 386 Department of Neuropathology, Neurological Institute, Kyushu University for their technical support 387 for the histopathological analysis. We also thank Akiko Yano, Miki Kijima and Mihoko Ohkubo for 388 their technical assistance. 


\section{References}

391 [1] Stromnes IM, Goverman JM. Active induction of experimental allergic encephalomyelitis. Nature protocols, 2006;1:1810-9.

393 [2] Stromnes IM, Goverman JM. Passive induction of experimental allergic encephalomyelitis. $394 \quad$ Nature protocols, 2006;1:1952-60.

395 [3] Komiyama Y, Nakae S, Matsuki T, Nambu A, Ishigame H, Kakuta S et al. IL-17 plays an important role in the development of experimental autoimmune encephalomyelitis. Journal of immunology, 2006;177:566-73. O'Connor RA, Prendergast CT, Sabatos CA, Lau CW, Leech MD, Wraith DC et al. Cutting edge: Th1 cells facilitate the entry of Th17 cells to the central nervous system during experimental autoimmune encephalomyelitis. Journal of immunology, 2008;181:3750-4. Oyamada A, Ikebe H, Itsumi M, Saiwai H, Okada S, Shimoda K et al. Tyrosine kinase 2 plays critical roles in the pathogenic CD4 T cell responses for the development of experimental autoimmune encephalomyelitis. Journal of immunology, 2009;183:7539-46. Smith CA, Gruss HJ, Davis T, Anderson D, Farrah T, Baker E et al. CD30 antigen, a marker for Hodgkin's lymphoma, is a receptor whose ligand defines an emerging family of cytokines with homology to TNF. Cell, 1993;73:1349-60. Shimozato O, Takeda K, Yagita H, Okumura K. Expression of CD30 ligand (CD153) on murine activated $\mathrm{T}$ cells. Biochemical and biophysical research communications, 1999;256:519-26.

Kennedy MK, Willis CR, Armitage RJ. Deciphering CD30 ligand biology and its role in humoral immunity. Immunology, 2006;118:143-52.

] Kim MY, Gaspal FM, Wiggett HE, McConnell FM, Gulbranson-Judge A, Raykundalia C et al. CD4(+)CD3(-) accessory cells costimulate primed CD4 T cells through OX40 and CD30 at sites where T cells collaborate with B cells. Immunity, 2003;18:643-54.

Sun X, Shibata K, Yamada H, Guo Y, Muta H, Podack ER et al. CD30L/CD30 is critical for maintenance of IL-17A-producing gammadelta T cells bearing Vgamma6 in mucosa-associated tissues in mice. Mucosal immunology, 2013;6:1191-201.

1] Shanebeck KD, Maliszewski CR, Kennedy MK, Picha KS, Smith CA, Goodwin RG et al. Regulation of murine B cell growth and differentiation by CD30 ligand. European journal of immunology, 1995;25:2147-53. costimulatory receptor CD30 is regulated by both CD28 and cytokines. Journal of immunology, 1998;160:2180-7. 
424

[13]

425

426

427

428

429

430

431

432

433

434

435

436

437

438

439

440

Lee SY, Lee SY, Kandala G, Liou ML, Liou HC, Choi Y. CD30/TNF receptor-associated factor interaction: NF-kappa B activation and binding specificity. Proceedings of the National Academy of Sciences of the United States of America, 1996;93:9699-703.

[14] Duckett CS, Gedrich RW, Gilfillan MC, Thompson CB. Induction of nuclear factor kappaB by the CD30 receptor is mediated by TRAF1 and TRAF2. Molecular and cellular biology, 1997; 17:1535-42.

[15] Wiley SR, Goodwin RG, Smith CA. Reverse signaling via CD30 ligand. Journal of immunology, 1996;157:3635-9.

[16] Sun X, Yamada H, Shibata K, Muta H, Tani K, Podack ER et al. CD30 ligand/CD30 plays a critical role in Th17 differentiation in mice. Journal of immunology, 2010;185:2222-30.

[17] Romagnani S, Del Prete G, Maggi E, Chilosi M, Caligaris-Cappio F, Pizzolo G. CD30 and type $2 \mathrm{~T}$ helper (Th2) responses. Journal of leukocyte biology, 1995;57:726-30.

[18] Bengtsson A. The role of CD30 in atopic disease. Allergy, 2001;56:593-603.

[19] Polte T, Behrendt AK, Hansen G. Direct evidence for a critical role of CD30 in the development of allergic asthma. The Journal of allergy and clinical immunology, 2006;118:942-8.

[20] Sun X, Somada S, Shibata K, Muta H, Yamada H, Yoshihara H et al. A critical role of CD30 ligand/CD30 in controlling inflammatory bowel diseases in mice. Gastroenterology, 2008;134:447-58.

[21] Tang C, Yamada H, Shibata K, Muta H, Wajjwalku W, Podack ER et al. A novel role of CD30L/CD30 signaling by T-T cell interaction in Th1 response against mycobacterial infection. Journal of immunology, 2008;181:6316-27.

[22] Fuchiwaki T, Sun X, Fujimura K, Yamada H, Shibata K, Muta H et al. The central role of $\mathrm{CD} 30 \mathrm{~L} / \mathrm{CD} 30$ interactions in allergic rhinitis pathogenesis in mice. European journal of immunology, 2011;41:2947-54.

[23] Zhang ZX, Yang L, Young KJ, DuTemple B, Zhang L. Identification of a previously unknown antigen-specific regulatory $\mathrm{T}$ cell and its mechanism of suppression. Nature medicine, 2000;6:782-9.

[24] Dai Z, Li Q, Wang Y, Gao G, Diggs LS, Tellides G et al. CD4+CD25+ regulatory T cells suppress allograft rejection mediated by memory CD8+ T cells via a CD30-dependent mechanism. The Journal of clinical investigation, 2004;113:310-7.

[25] Sun X, Yamada H, Shibata K, Muta H, Tani K, Podack ER et al. CD30 ligand is a target for a novel biological therapy against colitis associated with Th17 responses. Journal of immunology, 2010;185:7671-80.

[26] Blazar BR, Levy RB, Mak TW, Panoskaltsis-Mortari A, Muta H, Jones M et al. CD30/CD30 
ligand (CD153) interaction regulates CD4+ T cell-mediated graft-versus-host disease. Journal of immunology, 2004;173:2933-41.

[27] Nishimura H, Yajima T, Muta H, Podack ER, Tani K, Yoshikai Y. A novel role of CD30/CD30 ligand signaling in the generation of long-lived memory CD8+ T cells. Journal of immunology, 2005;175:4627-34.

[28] Veldhoen M, Hocking RJ, Flavell RA, Stockinger B. Signals mediated by transforming

[29] Bowen MA, Lee RK, Miragliotta G, Nam SY, Podack ER. Structure and expression of murine

\section{[30] Frentsch M, Arbach O, Kirchhoff D, Moewes B, Worm M, Rothe M et al. Direct access to} growth factor-beta initiate autoimmune encephalomyelitis, but chronic inflammation is needed

[32] Laurence A, Tato CM, Davidson TS, Kanno Y, Chen Z, Yao Z et al. Interleukin-2 signaling via

[33] Ransohoff RM, Kivisakk P, Kidd G. Three or more routes for leukocyte migration into the

[34] Tompkins SM, Padilla J, Dal Canto MC, Ting JP, Van Kaer L, Miller SD. De novo central

[35] Kivisakk P, Imitola J, Rasmussen S, Elyaman W, Zhu B, Ransohoff RM et al. Localizing of neuroantigen-specific $\mathrm{T}$ cells in the target organ determines the clinical outcome of autoimmune encephalomyelitis. The Journal of experimental medicine, 2004;199:185-97. central nervous system immune surveillance: meningeal antigen-presenting cells activate $\mathrm{T}$ cells during experimental autoimmune encephalomyelitis. Annals of neurology, 2009;65:457-69.

\section{Reboldi A, Coisne C, Baumjohann D, Benvenuto F, Bottinelli D, Lira S et al. C-C chemokine} receptor 6-regulated entry of TH-17 cells into the CNS through the choroid plexus is required for the initiation of EAE. Nature immunology, 2009;10:514-23.

8] Ruddle NH, Bergman CM, McGrath KM, Lingenheld EG, Grunnet ML, Padula SJ et al. An antibody to lymphotoxin and tumor necrosis factor prevents transfer of experimental allergic encephalomyelitis. The Journal of experimental medicine, 1990;172:1193-200. 
494 [39] Baker D, Butler D, Scallon BJ, O'Neill JK, Turk JL, Feldmann M. Control of established experimental allergic encephalomyelitis by inhibition of tumor necrosis factor (TNF) activity within the central nervous system using monoclonal antibodies and TNF receptor-immunoglobulin fusion proteins. European journal of immunology, 1994;24:2040-8. van Oosten BW, Barkhof F, Truyen L, Boringa JB, Bertelsmann FW, von Blomberg BM et al. Increased MRI activity and immune activation in two multiple sclerosis patients treated with the monoclonal anti-tumor necrosis factor antibody cA2. Neurology, 1996;47:1531-4.

[41] TNF neutralization in MS: results of a randomized, placebo-controlled multicenter study. The Lenercept Multiple Sclerosis Study Group and The University of British Columbia MS/MRI Analysis Group. Neurology, 1999;53:457-65.

504 [42] Nomura T, Abe Y, Kamada H, Shibata H, Kayamuro H, Inoue M et al. Therapeutic effect of PEGylated TNFR1-selective antagonistic mutant TNF in experimental autoimmune encephalomyelitis mice. Journal of controlled release : official journal of the Controlled Release Society, 2011;149:8-14.

[43] Brambilla R, Ashbaugh JJ, Magliozzi R, Dellarole A, Karmally S, Szymkowski DE et al. Inhibition of soluble tumour necrosis factor is therapeutic in experimental autoimmune encephalomyelitis and promotes axon preservation and remyelination. Brain : a journal of neurology, 2011;134:2736-54.

[44] Williams SK, Maier O, Fischer R, Fairless R, Hochmeister S, Stojic A et al. sclerosis. PloS one, 2014;9:e90117. 
519 Figure 1. CD30L KO mice are resistant to EAE.

520 (A) Clinical scores in WT and CD30L KO mice are shown. The numbers in parentheses express

521 disease incidence. Error bars represent mean \pm standard error of the mean. (B) Histopathological

522 analysis of spinal cords by hematoxylin and eosin staining was performed 18 days after immunization.

523 Right panels show the magnified view of the area within the square in left panels. Magnification, left,

$524 \times 40$; right, $\times 100$. (C) Klüver-Barrera's staining of spinal cord sections was performed on day 40 .

525 Arrowheads show demyelinated lesions. Magnification, $\times 40$. (D) The number of cells isolated from

526 spinal cords 18 days after immunization was calculated by flow cytometric analysis. Absolute cell

527 numbers of CD4+MHC Class II- (CD4 T cells, CD4), CD11b+MHC Class II+ (macrophage and

528 microglia, Mac/Mic), and Gr-1+MHC Class II- (granulocytes, Gr) cells in the spinal cord are shown.

529 Mean disease scores were $3.0(\mathrm{WT}, \mathrm{n}=4)$ and $2.25(\mathrm{CD} 30 \mathrm{~L} \mathrm{KO}, \mathrm{n}=4)$. Error bars represent mean \pm

530 standard deviation. Data are representative of three independent experiments. Statistical significance

531 was evaluated by Student's $t$-test. *, p $<0.05, * *, \mathrm{p}<0.01$.

532

Figure 2. CD30L/CD30 signaling contributes to antigen-specific proliferation and Th1 and Th17 
535 The dLNs cells were harvested on day 9 after EAE induction and restimulated in vitro with either

536 medium alone or indicated concentrations of $\mathrm{MOG}_{35-55}$ peptide. (A) Proliferation of dLNs cells in

537 response to $\mathrm{MOG}_{35-55}$ peptide was examined by thymidine incorporation assay. (B) The percentage and

538 number of $\mathrm{CD} 154^{+} \mathrm{CD} 4 \mathrm{~T}$ cells in dLNs induced by restimulation of $\mathrm{MOG}_{35-55}$ peptide. (C)

539 Intracellular cytokine staining of $\mathrm{CD} 4 \mathrm{~T}$ cells after culture with $\mathrm{MOG}_{35-55}$ peptide for 5 hours in the

540 presence of Brefeldin-A. (D) Concentrations of IL-17 and IFN- $\gamma$ measured by ELISA in the culture

541 supernatant of dLNs cells $\left(5 \times 10^{5}\right)$ after restimulation by $\mathrm{MOG}_{35-55}$ peptide for 3 days. (E) Cytokine

542 production of IL-17 and IFN- $\gamma$ by CD4 T cells co-cultured with APCs from WT or CD30L KO mice.

543 Purified WT CD4 T cells $\left(2 \times 10^{5}\right)$ were cultured with 30 Gy-irradiated WT or CD30L KO splenocytes

544 in the medium with or without $\mathrm{MOG}_{35-55}$ peptide for 72 hours. The concentrations of IL-17 and IFN- $\gamma$

545 were measured by ELISA. Data are representative of three independent experiments. Error bars

546 represent mean \pm standard deviation. Statistical significance was evaluated by Student's $t$-test. * $\mathrm{p}<$

$547 \quad 0.05, * * \mathrm{p}<0.01$

548

549 Figure 3. CD30L on hematopoietic cells and radioresistant cells is involved in the development of

550 EAE. 
551 (A) Three sets of BM chimera were prepared as follows: WT (Ly5.1/5.1) to WT (Ly5.2/5.2), WT

552 (Ly5.1/5.1) to CD30L KO (Ly5.2/5.2), and CD30L KO (Ly5.2/5.2) to WT (Ly5.1/5.1). Recipient mice

553 were lethally irradiated and transplanted with BM from donor mice. Active EAE was induced after

554 confirming BM reconstitution 8 weeks later. (B) Clinical courses and (C) body weight changes are

555 shown. The numbers in parentheses express disease incidence. Data are representative of two different

556 experiments. Error bars represent mean \pm standard error of the mean. Statistical differences were

557 evaluated by Dunnett's test for multiple comparisons to control of WT -> WT chimera. *, p $<0.05$

558 (WT- $>$ CD30L KO chimera) $; \uparrow, \mathrm{p}<0.05 ; \dagger \uparrow, \mathrm{p}<0.01(\mathrm{CD} 30 \mathrm{~L}$ KO $->$ WT chimera).

560 Figure 4. CD30L KO mice are resistant to passive EAE.

561 The dLNs cells were harvested from WT mice 10 days after EAE induction and restimulated in vitro

562 with $\mathrm{MOG}_{35-55}$ peptide and recombinant mouse IL-23 for 3 days. Subsequently, cultured cells were

563 intravenously transferred into 4 Gy-irradiated naive WT or CD30L KO mice $\left(2 \times 10^{7}\right.$ cells per

564 recipient) followed by i.p. injection of PTX on day 0 and 2 after the transfer. (A) Clinical scores and

565 (B) body weight changes are shown. The numbers in parentheses express disease incidence. (C)

566 Histopathological analysis of spinal cords was performed on day 40 after the transfer by hematoxylin

567 and eosin staining. Lower panels show a magnified view of the area within the square in upper panels. 
568 Magnification, upper, $\times 40$; lower, $\times 100$. (D) Klüver-Barrera's staining was performed on day 40 after

569 the transfer. Arrowheads show demyelinated lesions. Magnification, $\times 40$. Data are representative of

570 three independent experiments. Error bars represent mean \pm standard SEM. Statistical significance was

571 evaluated by Student's $t$-test. *, $\mathrm{p}<0.05$.

572

573 Figure 5. Soluble mCD30-Ig treatment at the induction phase ameliorates actively induced EAE.

574 (A) Active EAE was induced in WT mice and they received $200 \mu \mathrm{g}$ of mCD30-Ig or PBS

575 intraperitoneally on days 0,2, 4, 6 and 8. (B) Clinical courses and (C) body weight changes are shown.

576 The numbers in parentheses express disease incidence. Error bars represent mean \pm standard error of

577 the mean. (D) Histopathological analysis of spinal cords by hematoxylin and eosin staining were

578 performed on day 17. Lower panels show the magnified view of the area within the square in upper

579 panels. Magnification, upper, $\times 40$; lower, $\times 100$. (E) dLNs cells harvested on day 9 were restimulated

580 in vitro with $\mathrm{MOG}_{35-55}$ peptide for 5 hours in the presence of brefeldin-A. The expression of $\mathrm{CD} 154$

581 and (F) intracellular cytokine staining of CD4 T cells were examined by flow cytometry. Error bars

582 represent mean \pm standard deviation. Data are representative of three independent experiments.

583 Statistical significance was evaluated by Student's $t$-test. *, p $<0.05 ; * *, \mathrm{p}<0.01$. 
585 Figure 6. Soluble mCD30-Ig treatment after disease onset ameliorates EAE.

586 (A) Active EAE was induced in WT mice and they were administered $200 \mu \mathrm{g}$ of mCD30-Ig or PBS i.p.

587 on days $12,14,16,18$ and 20. (B) Clinical courses and (C) body weight changes are shown. The

588 numbers in parentheses express disease incidence. Error bars represent mean \pm standard error of the

589 mean. (D) Histopathological analysis of spinal cords was performed by hematoxylin and eosin

590 staining on day 30. Lower panels show the magnified view of the area within the square in upper

591 panels. Magnification, upper, $\times 40$; lower, $\times 100$. (E) Klüver-Barrera's staining was also performed on

592 day 30. Arrowheads show demyelinated lesions. Magnification, $\times 40$. Data are representative of three

593 independent experiments. Statistical significance was evaluated by Student's $t$-test. *, p < $0.05 ; * *, \mathrm{p}<$

5940.01.

595

596 
Figure 1

A
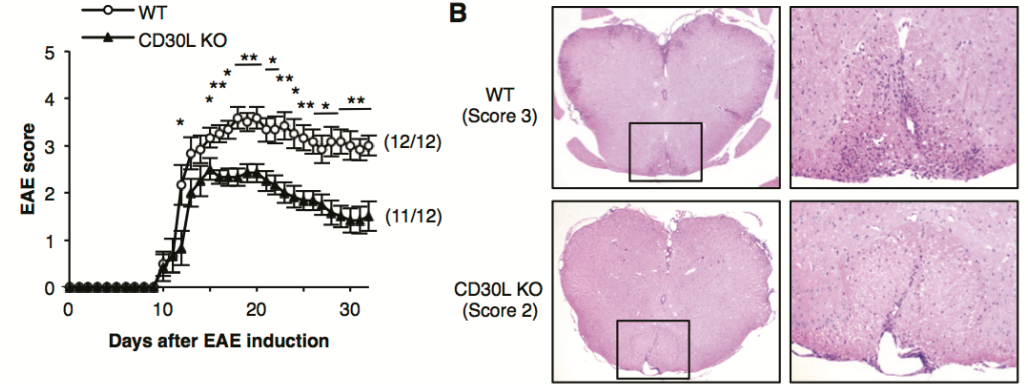

c

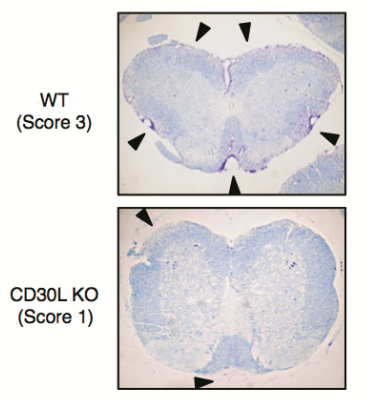

D

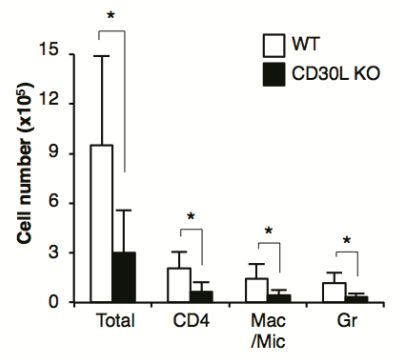


Figure 2

A

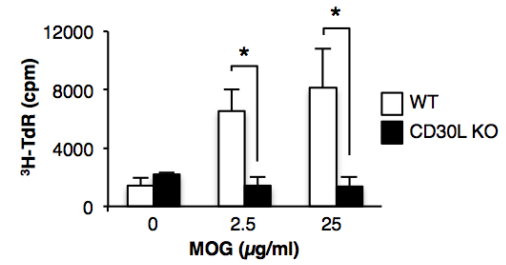

B
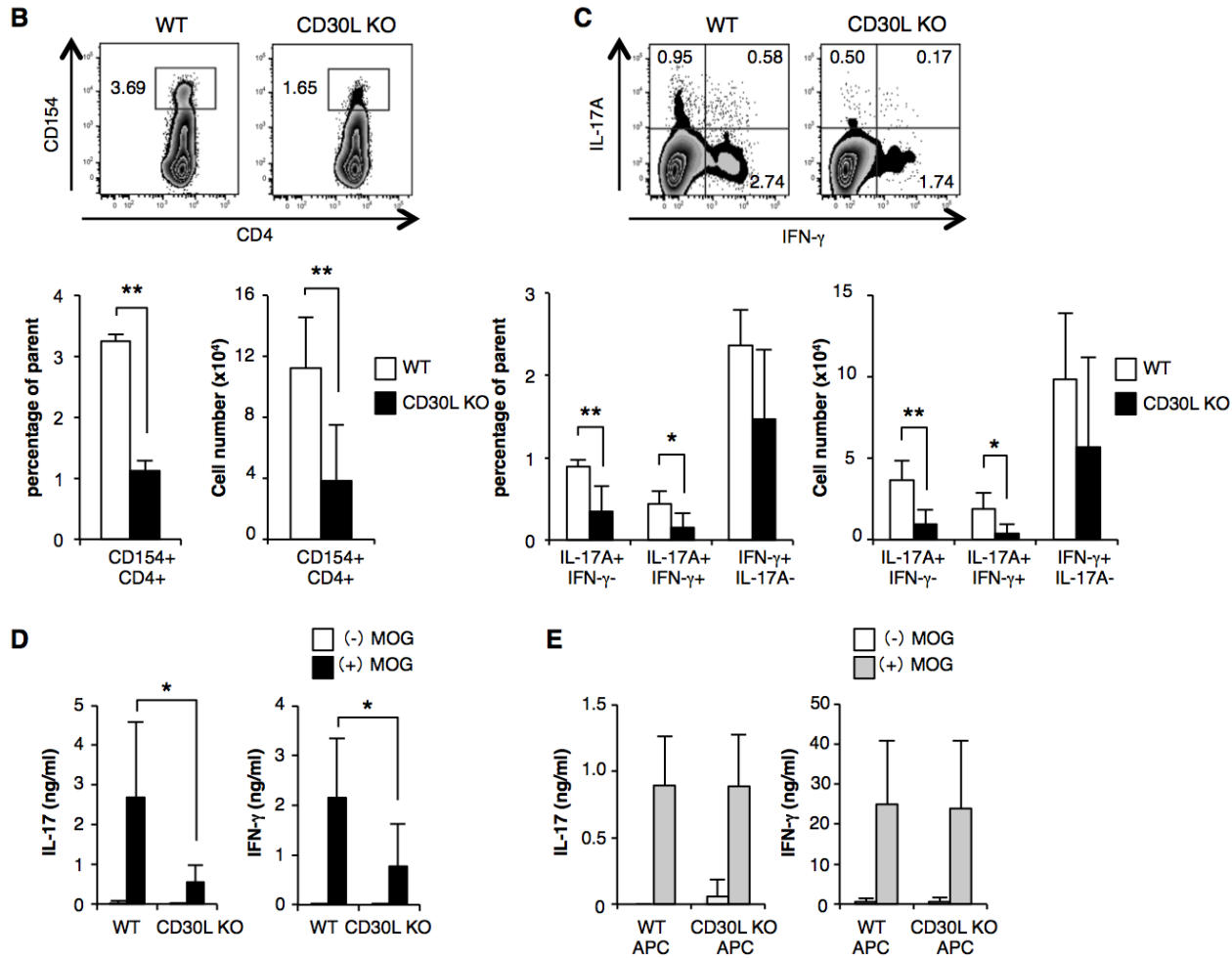
Figure 3

A

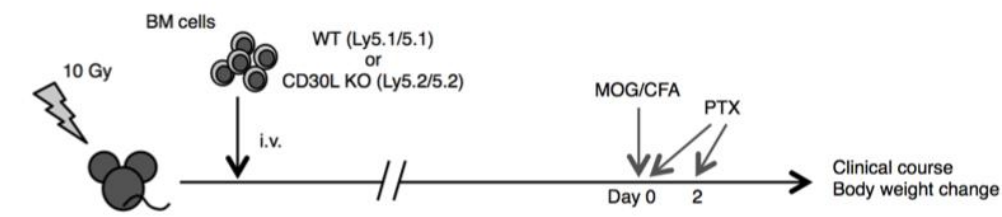

WT (Ly5.1/5.1 or Ly5.2/5.2)

CD30L KO(Ly5.2/5.2)

B $\quad \rightarrow W T \rightarrow W T \quad(10 / 10)$

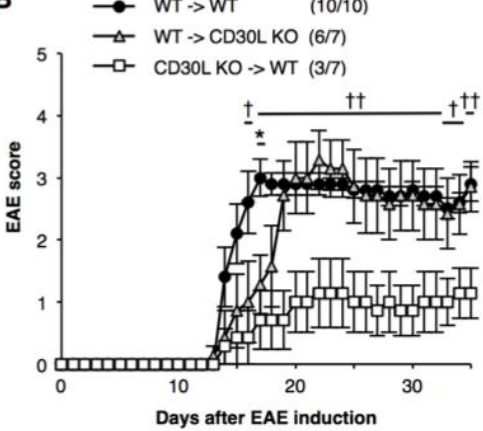

C

$\rightarrow W T \rightarrow W T$

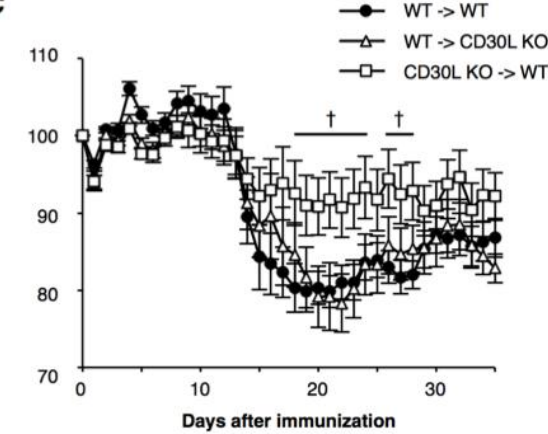


Figure 4

A

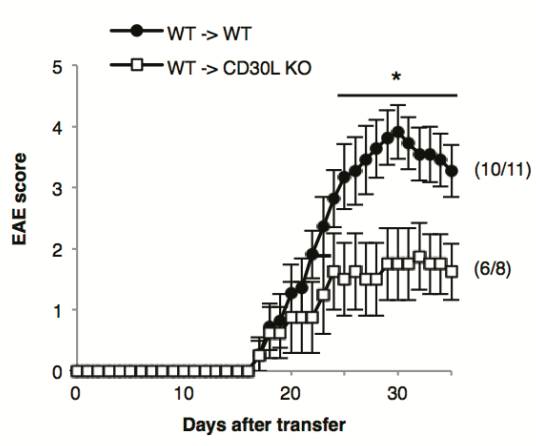

B

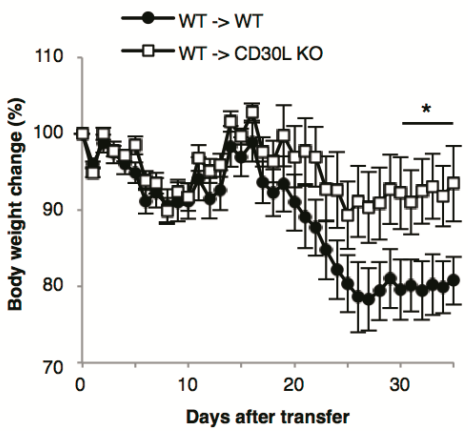

C

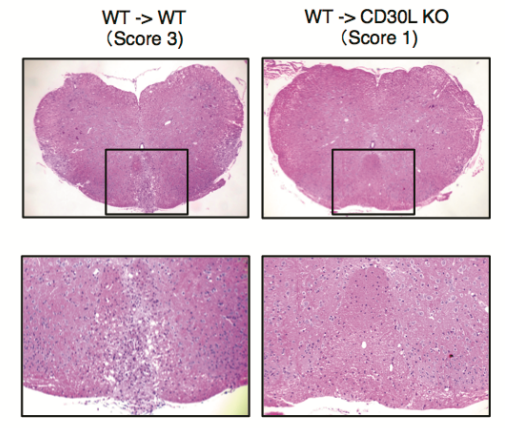

D

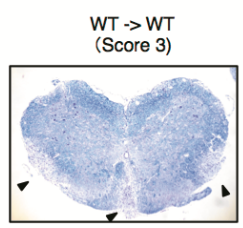

WT $\rightarrow$ CD3OL KO (Score 1)

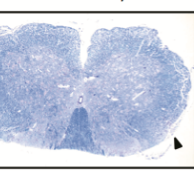




\section{Figure 5}

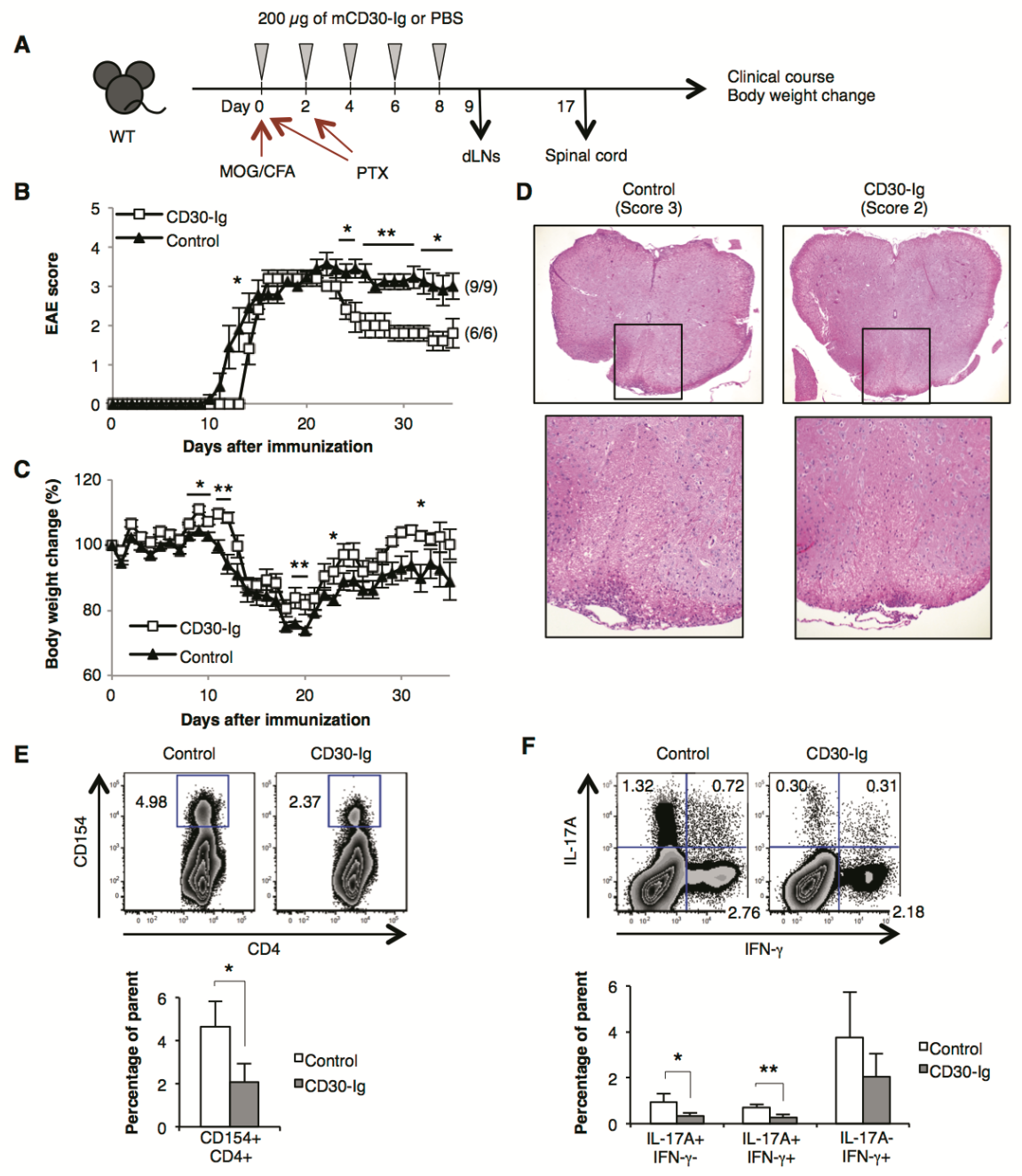


Figure 6

A

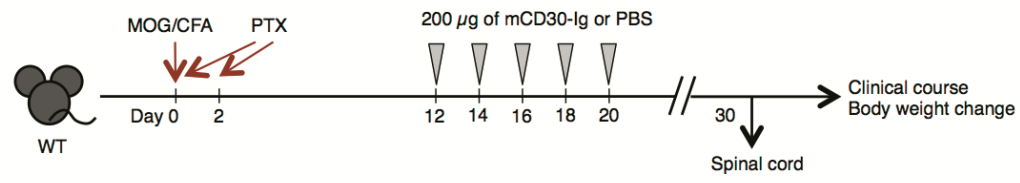

B

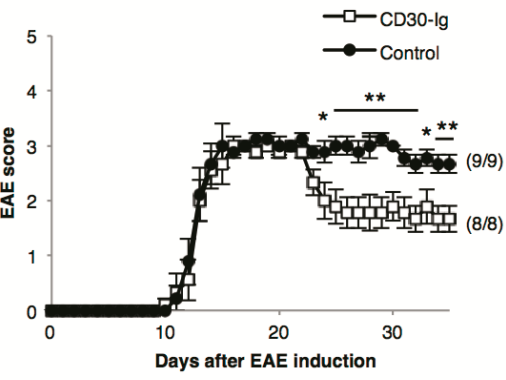

D

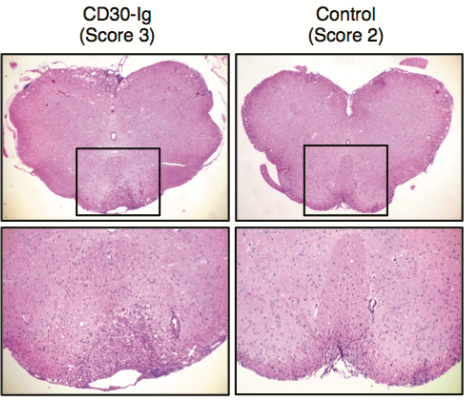

C

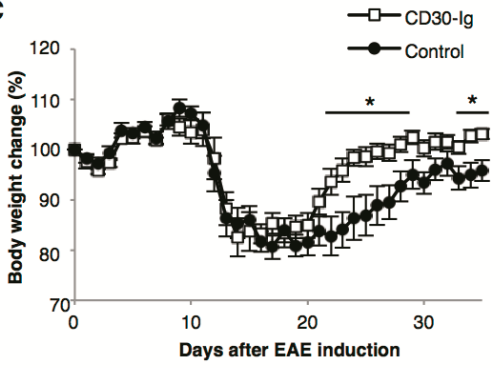

E

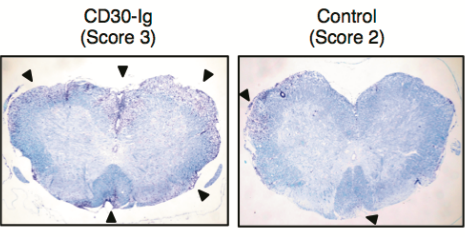

